\title{
Modeling the photometric and dynamical behavior of Super-Schmidt meteors in the Earth's atmosphere
}

\author{
L. R. Bellot Rubio ${ }^{1,4}$, M. J. Martínez González ${ }^{2}$, L. Ruiz Herrera ${ }^{2}$, J. Licandro ${ }^{3}$, \\ D. Martínez Delgado ${ }^{4}$ P. Rodríguez Gil ${ }^{4}$, and M. Serra-Ricart ${ }^{4}$ \\ 1 Kiepenheuer-Institut für Sonnenphysik, Schöneckstr. 6, 79104, Freiburg, Germany \\ 2 Dept. de Astrofísica, Universidad de La Laguna, La Laguna, Tenerife, Spain \\ 3 Centro Galileo Galilei and Telescopio Nazionale Galileo, La Palma, Spain \\ ${ }^{4}$ Instituto de Astrofísica de Canarias, 38200, La Laguna, Tenerife, Spain
}

Received 15 March 2002 / Accepted 29 April 2002

\begin{abstract}
Faint meteors observed with Super-Schmidt cameras are re-examined in order to assess whether their dynamical and photometric behavior can be described by means of the single body theory. Velocities, decelerations and magnitudes are fitted simultaneously to synthetic curves resulting from integration of the appropriate set of differential equations. The parameters determined by this procedure are the ablation coefficient, the shape-density coefficient and the preatmospheric mass of each individual meteoroid. It turns out that $73 \%$ of the meteors analyzed here (with magnitudes in the range from +2.5 to -5 ) are reasonably well described by this theory, suggesting that they did not undergo significant fragmentation during their atmospheric flight. Nevertheless, we identify some systematic differences between observed and theoretical light curves of meteors for which the fit is good. Meteoroid bulk densities are estimated from the retrieved shape-density coefficients. The distributions of individual values are broad, indicating that objects of different densities coexist within the same meteoroid population. The average density is found to be 2400, 1400, and $400 \mathrm{~kg} \mathrm{~m}^{-3}$ for A-type, B-type and C-type meteoroids, respectively. These results do not confirm the large values determined from quasicontinuous fragmentation models.
\end{abstract}

Key words. meteors, meteoroids

\section{Introduction}

Our capability to model the interaction between the Earth's atmosphere and meteoroids, particularly the smaller ones, is rather limited. As a consequence, their physical properties remain uncertain. This is especially true for some important quantities, such as meteoroid bulk densities. Early in the 1950s it was recognized that the instantaneous dynamical and photometric masses of faint meteors may differ by an order of magnitude as determined from the observed decelerations and the light curve, respectively. The computation of dynamical masses requires knowledge of the meteoroid bulk density, while that of photometric masses needs very accurate luminous efficiencies. At the time, none of these quantities were known with precision. On the assumption of equal photometric and dynamical masses on average, Verniani (1965) derived a mean density of $250 \mathrm{~kg} \mathrm{~m}^{-3}$ for all observed meteors. Ceplecha (1966) pointed out that Verniani's value was in error because of the assumption that all meteoroids share

Send offprint requests to: L. R. Bellot Rubio, e-mail: lbellot@kis. uni-freiburg.de the same properties. Indeed, he demonstrated in a convincing way that there exist several meteoroid populations characterized by slightly different physical properties (composition, structure, and bulk density). Distinct meteoroid populations were first recognized by Jacchia (1958) and Ceplecha (1958) on the basis of differences in the beginning heights of the luminous meteor trajectories. Ceplecha $(1967,1968)$ proposed to use the observed beginning heights to assign mean densities to particles belonging to the different meteoroid populations. This made it possible to determine more realistic meteoroid bulk densities, which turned out to be larger than Verniani's value. In particular, A-type and C-type meteoroids were ascribed mean densities of $2000 \mathrm{~kg} \mathrm{~m}^{-3}$ and $750 \mathrm{~kg} \mathrm{~m}^{-3}$, respectively (Ceplecha et al. 1998).

Also in the 1950s another piece of evidence emerged from the analysis of Super-Schmidt meteors. Jacchia (1955) found that the dynamical mass of some faint meteors decreases more rapidly than the photometric mass as the particle moves along its atmospheric trajectory. In order to account for these anomalous decelerations, he introduced the concept of progressive fragmentation, whereby 
meteoroids fragment into smaller pieces during the atmospheric flight. Visible forms of fragmentation such as wake and terminal blending can sometimes be recognized in the light curves of faint meteors showing deceleration anomalies. In general, these meteors are shorter and tend to reach maximum brightness at higher heights than normal meteors.

The fragmentation theory soon gained popularity. A general model of quasi-continuous fragmentation (i.e., the evaporation of solid particles continuously detached from the main body) was worked out by Novikov et al. (1984) and extended by Babadzhanov et al. (1988). According to Babadzhanov (1992), about one third of the faint meteoroids undergo quasi-continuous fragmentation (hereafter QCF). Several methods were suggested to determine the parameters describing the QCF behavior of meteoroids, including the comparison of theoretical and observed heights and the fitting of light curves. Already the earliest applications of the QCF model yielded extremely high meteoroid bulk densities. Lebedinets (1987) and Babadzhanov (1994), for example, obtained an average density of $3300 \mathrm{~kg} \mathrm{~m}^{-3}$, with values for individual meteoroids ranging from 100 to $8000 \mathrm{~kg} \mathrm{~m}^{-3}$. If such large values are real, most meteoroids would be iron or stony particles. This is in contradiction with the results described above.

Meteoroid bulk densities are important because of their implications for the composition and structure of cometary nuclei, and also for estimating the meteoroid mass influx to the Earth. The present situation, in which very different bulk densities result depending on the adopted scenario, is rather unsatisfactory. We believe that part of the discrepancy is due to the fact that specific ablation modes are assumed a priori, instead of using all the information encoded in decelerations and light curves to determine the real ablation mode of individual meteoroids.

In our opinion, the first step toward obtaining reliable bulk densities is to give a precise answer to the question of whether fragmentation is a common process or not. In other words, we must determine the fraction of meteors whose behavior can only be understood in terms of fragmentation. Fragmentation has a big impact on the dynamical and photometric signatures of meteoroids. However, using observed light curves as the sole diagnostics may lead to wrong results because there is no guarantee that a model which is able to reproduce the photometric behavior of a given meteoroid can also provide a good description of its dynamical behavior.

Because of these reasons, we attempt here a simultaneous modeling of the dynamical and photometric behavior of faint meteors by means of a least-squares procedure. We examine the simplest scenario, i.e., no fragmentation. Brighter meteors than those considered in this work have been shown to behave as compact, nonfragmenting particles (e.g. McCrosky \& Ceplecha 1970). Therefore, it seems natural to check the validity of this ablation mode for the fainter Super-Schmidt meteors. Earlier attempts in this direction were simplistic by necessity. Now we have a much better knowledge of luminous efficiencies, for instance, and present-day computers make it possible to carry out extensive numerical calculations taking into account all available data. An appropriate description of the behavior of nonfragmenting meteoroids is provided by the single body theory (see McCrosky \& Ceplecha 1970 and Brohnshten 1983).

Considering that many faint meteors do not show anomalies in their light curves, we may anticipate that a significant fraction of all meteoroids indeed conform to this model. Meteors that cannot be described in the framework of the single body theory probably undergo fragmentation processes. Our analysis will then give a precise answer to the question raised above. Moreover, it will allow us to estimate the bulk densities and ablation parameters of those meteoroids whose behavior is not severely influenced by fragmentation processes.

Preliminary results of this investigation have been published by Bellot Rubio et al. (2001).

\section{Observations}

The observational material used here is the catalog of very precise photographic Super-Schmidt meteors of Jacchia et al. (1967, hereafter JVB). Among the various sources available for faint meteors, this compilation is generally regarded as being the most accurate. Another advantage is that it has been employed in many investigations, so our using it will allow direct comparisons with previously published results.

JVB catalog contains precise velocity and deceleration measurements for 413 faint meteors spanning a magnitude range between +2.5 and -5 mag. These meteors are indeed a subset of all meteors photographed in New Mexico with the $0.3 \mathrm{~m}, \mathrm{f} / 0.8$ Super-Schmidt cameras of the Harvard Meteor Project (McCrosky \& Posen 1961). In order to compute very accurate decelerations from the photographic records, JVB selected those meteors having the longer trajectories. Hence, JVB material cannot be considered as a random sample. In particular, the percentage of particles belonging to the different meteoroid populations is different compared to the more homogeneous, complete Super-Schmidt database.

The classification of meteoroids into four populations (A-group comprising particles similar to carbonaceous chondrites; B-group of dense cometary material; Cgroup of regular cometary material; and D-group of soft cometary material) is accomplished by means of the $K_{\mathrm{B}}$ parameter. It is defined as

$K_{\mathrm{B}}=\log \rho_{\mathrm{B}}+2.5 \log v_{0}-0.5 \log \cos z_{\mathrm{R}}$,

where $\rho_{\mathrm{B}}$ is the air density at the beginning of the luminous trajectory (in $\mathrm{g} \mathrm{cm}^{-3}$ ), $v_{0}$ is the preatmospheric velocity of the meteoroid (in $\mathrm{cm} \mathrm{s}^{-1}$ ), and $z_{\mathrm{R}}$ is the inclination of the trajectory with respect to the vertical. According to Ceplecha (1988), the range of $K_{\mathrm{B}}$ values identifying each population is $7.3 \leq K_{\mathrm{B}}<8.0$ (A group), 
$7.1 \leq K_{\mathrm{B}}<7.3$ (B group), $6.6 \leq K_{\mathrm{B}}<7.1$ (C group) and $K_{\mathrm{B}}<6.6$ (D group).

While the complete Super-Schmidt sample contains $50 \%$ and $45 \%$ of A-type and C-type meteoroids, respectively, the percentage of particles belonging to these groups in JVB list is $32 \%$ and $62 \%$ (Ceplecha 1988). Hence, low-density particles are overrepresented in our material, and this will have to be considered when interpreting the results of the analysis.

For each meteor, JVB's catalog provides us with precise measurements of the instantaneous velocity and deceleration at several points along the atmospheric trajectory (i.e., at different heights above the Earth's surface). The catalog also gives the observed light curve by specifying the absolute magnitude of the meteor at four different heights: the beginning and end of the trajectory, the point of maximum light, and the point where the brightening meteor reached an absolute photographic magnitude of +2.5 . The smooth light curves of these faint meteors in the original plates imply that they can be described well with only four points. For various reasons, the photometry of photographic meteors is of much lower quality than velocity and deceleration measurements. This translates into uncertainties of up to $\pm 0.5 \mathrm{mag}$ in the light curves. However, such uncertainties are still appropriate to distinguish meteors showing abnormal photometric behaviors.

We have selected 370 out of the 413 meteors in JVB catalog. The remainder were not considered because their velocities, decelerations or magnitudes could be measured at only one point along the trajectory. This leaves us with $90 \%$ of the original data, but we ensure the high quality of the material selected for treatment. Our sample include 285 sporadic and 85 shower meteors.

\section{The model}

In order to check whether faint meteors behave as nonfragmenting particles, we model their interaction with the atmosphere by means of the single body theory (e.g., Bronshten 1983). The motion and ablation of a compact, nonfragmenting object are described by the drag and mass-loss equations

$$
\begin{aligned}
& \frac{\mathrm{d} v}{\mathrm{~d} t}=-K \rho m^{-1 / 3} v^{2}, \\
& \frac{\mathrm{d} m}{\mathrm{~d} t}=-\sigma K \rho m^{2 / 3} v^{3},
\end{aligned}
$$

where $t$ is time, $v$ the instantaneous velocity, $m$ the instantaneous mass, $\rho$ the air density, $\sigma$ the ablation coefficient, and $K$ the shape-density coefficient. By adopting Eq. (3) we make the assumption that intensive evaporation has already started. Jones \& Kaiser (1966) pointed out that the early parts of the light curve may be inhibited as the finite heat capacity of the meteoroid material results in heating without intensive ablation. Recent analyses (e.g. Fisher et al. 2000) explicitly allow for two regimes to partially account for this effect.
The height in the atmosphere $h$ is related to $t$ by the geometrical relationship $\mathrm{d} h / \mathrm{d} t=v \cos z$, where $z$ is the inclination of the trajectory with respect to the vertical. For simplicity, we assume constant ablation and shapedensity coefficients. These parameters depend on the physical properties of the meteoroids according to

$$
\begin{aligned}
& \sigma=\frac{\Lambda}{2 \Gamma Q}, \\
& K=\frac{\Gamma A}{\delta^{2 / 3}},
\end{aligned}
$$

where $\Lambda$ is the heat-transfer coefficient, $\Gamma$ the drag coefficient, $Q$ the heat of ablation, $\delta$ the meteoroid bulk density, and $A$ is called the shape factor. $A=S / V^{2 / 3}$, with $S$ and $V$ the meteoroid's cross section and volume, respectively, is a measure of the frontal area of the particle. For faint meteors such as those analyzed in this work we may assume free molecular flow, hence $\Gamma=1$.

The photometric behavior of nonfragmenting particles is described by the luminous equation

$I=\frac{\tau}{2}\left(-\frac{\mathrm{d} m}{\mathrm{~d} t}\right) v^{2}$,

with $I$ the radiation intensity and $\tau$ the so-called luminous efficiency. This expression is derived on the assumption that the intensity emitted by the meteoroid is proportional to the kinetic energy of the ablated material. As pointed out by McCrosky \& Ceplecha (1970), the simple form of Eq. (6) is the result of a severe lack of knowledge of the complex processes leading to light emission.

The luminous efficiency is a fundamental quantity because it makes it possible to determine the mass of the meteoroid from the light curve. In order to avoid big errors, very precise values of $\tau$ have to be used. A simplified functional dependence of the form $\tau=\tau_{0} v$ has often been adopted in earlier analyses. Jacchia et al. (1967), for example, used such a dependence with $\tau_{0}=$ $10^{-1} 0$ mag kg-1 $\mathrm{km}^{-3} \mathrm{~s}^{4}$ (for $v$ in $\mathrm{km} \mathrm{s}^{-1}$, these units imply that $I$ is expressed in terms of the intensity of a 0 mag star, so the absolute magnitude can be computed as $M=-2.5 \log I$ ). Recently, ReVelle \& Ceplecha (2001) have obtained very accurate luminous efficiencies from an in-depth analysis of a large sample of meteors. Since realistic values are crucial, we adopt their expressions in the hope that this will significantly improve our results. More specifically, we take

$$
\begin{array}{rlrl}
\ln \tau= & -2.338+\ln v+1.15 \tanh (0.38 \ln m) & v \geq 25.372 \\
\ln \tau= & -0.344-10.307 \ln v+9.781 \ln ^{2} v- & \\
& -3.0414 \ln ^{3} v+0.3213 \ln ^{4} v+ & \\
& +1.15 \tanh (0.38 \ln m) & & v<25.372
\end{array}
$$

where $\tau$ is expressed in percent, $v$ in $\mathrm{km} \mathrm{s}^{-1}$ and $m$ in $\mathrm{kg}$. This luminous efficiency has to be multiplied by $6.5313 \times$ $10^{-13}$ in order for the intensity to be expressed in units of a 0 mag star. Such a conversion factor holds for an average temperature of $4500 \mathrm{~K}$ (Ceplecha et al. 1998). 


\section{Numerical procedure}

Equations (2) and (3) form a system of ordinary differential equations. Synthetic curves $v=v(h), \mathrm{d} v / \mathrm{d} t=$ $\mathrm{d} v / \mathrm{d} t(h)$ and $I=I(h)$ are constructed by integrating them for given initial values of $v$ and $m$, and parameters $\sigma$ and $K$. The theoretical curves are then compared with the observed velocities, decelerations, and magnitudes in order to determine the best-fitting $\sigma$ and $K$ coefficients.

For the integration of Eqs. (2) and (3) we use a fourth-order Runge-Kutta method with constant step size $\Delta h=100 \mathrm{~m}$. This step size is appropriate even for the very few high velocity meteoroids present in the sample. $\sigma$ and $K$ are assumed to be constant during the atmospheric flight. There is strong evidence for differential ablation in meteor trails (e.g., von Zahn et al. 1999), implying that one actually needs a theory capable of accounting for the different chemical species separately. Since such models have not been developed so far, it seems reasonable to follow the usual procedure and adopt a single ablation parameter $\sigma$ for the whole trajectory. For simplicity, the air density at $h$ is computed by interpolation of the US Standard Atmosphere 1976. We have checked that solar-cycle related, latitude, and seasonal variations of the atmospheric density do not modify the results of the analysis to a large extent. This has been verified by using the more complex NASA's Mass-SpectrometerIncoherent-Scatter Extended (MSISE) 1990 atmospheric model (Hedin 1991) for a sample of random meteoroids ${ }^{1}$. The preatmospheric velocity and initial mass of each meteoroid are taken from JVB's catalog. The initial mass is photometric (obtained by integration of the light curve with $\tau_{0}=10^{-1} 0 \mathrm{mag} \mathrm{kg} \mathrm{km}^{-1} \mathrm{~s}^{4}$ ), and therefore not very reliable given the uncertainties in $\tau$.

The experimental data $(v, \mathrm{~d} v / \mathrm{d} t$, and magnitude as functions of $h$ ) are fitted to the synthetic curves by means of a non-linear, least-squares Marquardt algorithm (Press et al. 1986). The quantity minimized during the fit is

$\chi^{2}=\frac{1}{N-m} \sum_{i=1, N}\left[\frac{y_{i}^{\mathrm{obs}}-y_{i}^{\mathrm{synt}}}{\sigma_{i}} w_{i}\right]^{2}$,

where $N$ represents the number of observables, $m$ the number of free parameters, $y^{\text {obs }}$ and $y^{\text {synt }}$ the observed and synthetic quantities, respectively, $\sigma_{i}$ the uncertainty of $y_{i}^{\text {obs }}$, and $w_{i}$ a weight introduced to force better fits to selected observables. The free parameters determined from the inversion are $\sigma, K$, and the initial mass $m_{0}$. The partial derivatives of $v, \mathrm{~d} v / \mathrm{d} t$, and magnitude with respect to the free parameters are computed numerically. The weights $w$

\footnotetext{
${ }^{1}$ The maximum differences in the best-fitting $K$ and $\sigma$ parameters resulting from both atmospheric models are $15 \%$ and $9 \%$, respectively. Such values, however, are very seldomly encountered. The actual differences for most meteoroids are smaller than $5 \%$ and $3 \%$, respectively. We note that use of the MSISE model does not lead to better fits, only to slightly different $K$ and $\sigma$ parameters
}

assigned to velocities, decelerations, and magnitudes are $2,0.5$, and 1 , respectively. By this strategy we ensure that velocities (the best-known observables) are reproduced to a higher degree of precision than the other measurements. Additionally, given the importance of the light curve for constraining the free parameters, we force better fits to magnitudes than to decelerations. In any case, we have confirmed that the exact choice of weights does not significantly modify the final parameters. The solution appears to be largely unique for good fits, implying that there is just one combination of free parameters capable of explaining the observations regardless of the weights used to find it.

\section{Results}

Figure 1 shows three examples of meteors for which the single body theory provides an excellent description of their dynamical and photometric behavior. Not only the velocities and decelerations are well fitted, but also the light curves. To our knowledge, this is the first time that both dynamical and photometric data are reproduced within the error bars of the observations. The excellent fits presented in Fig. 1 strongly supports the view that these meteoroids did not undergo significant fragmentation during their atmospheric passage.

All in all, 270 out of the 370 meteors examined (i.e., $73 \%$ ) are reasonably well described by the single body theory ${ }^{2}$. The degree of success of this model is similar for sporadic $(72 \%)$ and shower $(78 \%)$ meteors. Table 1 summarizes the fraction of meteors that can be regarded as compact, nonfragmenting objects, for the various meteoroid populations. The most obvious result from Table 1 is that less than half of the A-type meteoroids conform to the single body theory. By contrast, $68 \%$ of the B-type and $86 \%$ of the C-type meteoroids appear to be nonfragmenting particles. This is quite an unexpected finding, as A-type meteoroids are believed to be the densest particles among the four groups considered in Table 1. Our results seems to imply that fragmentation processes are more efficient for the denser meteoroids.

At this point it is important to mention that, even in the case of good fits, the photometric behavior is somewhat difficult to reproduce. In particular, we find the following general trends:

\section{- The brightest magnitude is usually $0.2-0.5$ mag fainter than observed; \\ - The predicted height of maximum light is often higher than the observed height by $2-5 \mathrm{~km}$; \\ - The magnitude at the beginning point is usually brighter than observed.}

\footnotetext{
${ }^{2}$ Good fits are identified mainly on the basis of the final $\chi^{2}$ value, but the shapes of the synthetic curves have also been considered. In general, the single body theory is deemed appropriate for a meteor if $\chi^{2}$ is smaller than 10 .
} 

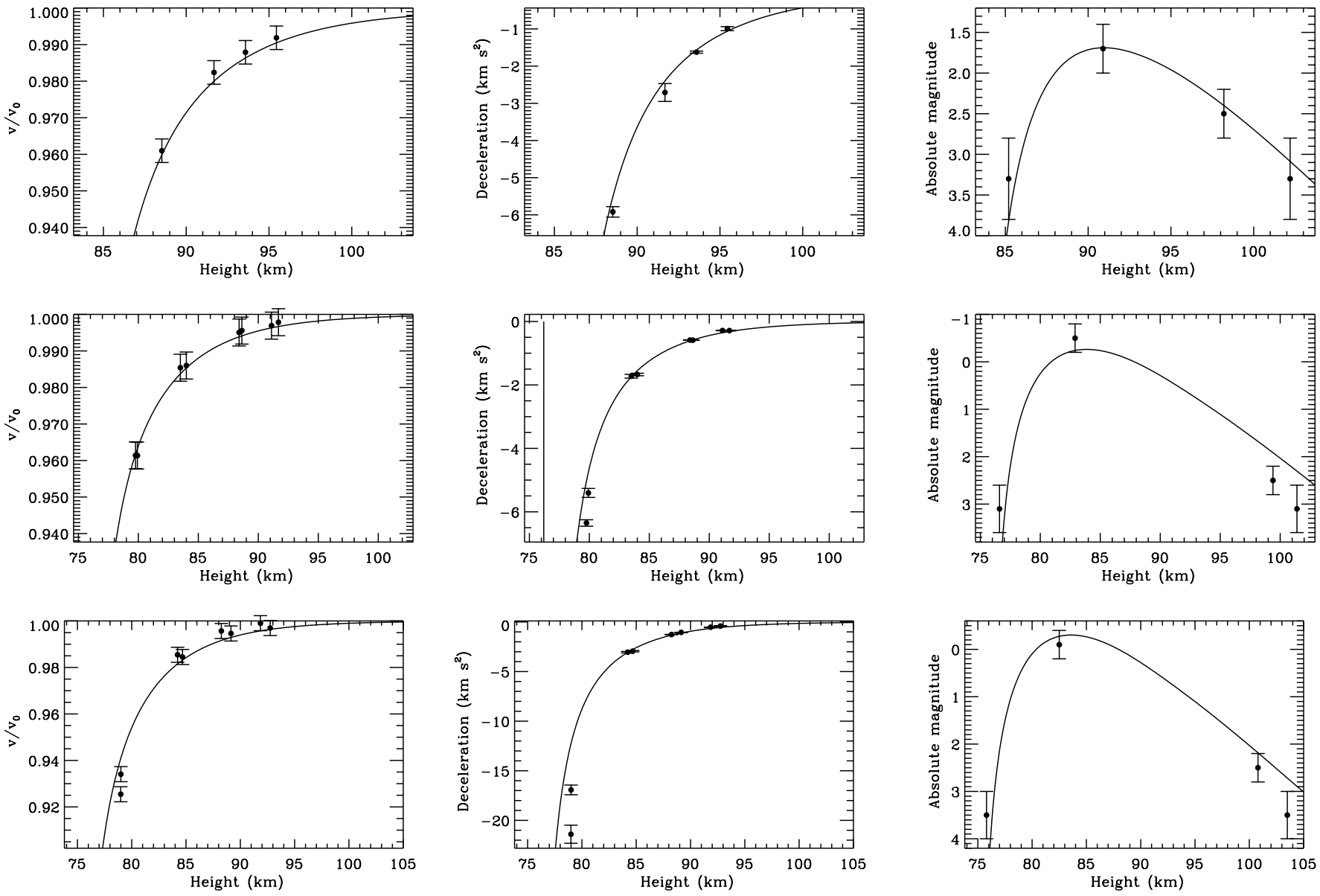

Fig. 1. Example of the application of the single body theory to faint Super-Schmidt photographic meteors. Experimental velocities, decelerations, and magnitudes (dots) are plotted in the left, central, and right panels, respectively, for meteors 8945 (top), 6949 (middle), and 9015 (bottom) in JVB's catalog. The solid lines show the best-fit curves resulting from the inversion of the observed quantities. The bulk densities of these meteoroids are found to be 300,1000 , and $600 \mathrm{~kg} \mathrm{~m}^{-3}$, respectively.

These systematic differences might be produced by fragmentation. However, the excellent fit to velocities and decelerations suggests that fragmentation processes, if any, have a minor influence on the observed quantities.

The behavior of 100 meteors $(28 \%)$ in our sample cannot be explained in terms of the ablation of nonfragmenting particles with constant $\sigma$ and $K$ parameters. For these meteors, the theoretical light curves differ significantly from the observed ones, and sometimes the dynamical data are also badly fitted. An example is given in Fig. 2, where we plot the observed and best-fit velocities, decelerations and magnitudes of the sporadic, A-type meteoroid 4141. Such a failure of the single body theory might be remedied by allowing the ablation and shape-density coefficients to change along the trajectory, in a similar way as Ceplecha et al. (2000). However, the large misfits suggest that these meteoroids indeed underwent other processes whose proper description would require a different model. In the remainder of the paper, we will therefore concentrate on those meteors for which the single body theory is valid.

\subsection{Ablation coefficients}

The ablation coefficient $\sigma$ governs the mass loss rate during the atmospheric flight. This parameter has a major impact on the light curve (see Eqs. (3) and (6)), but it also influences the motion of the particle through $m$ (Eq. (2)). We want to stress here that the product $\tau \sigma$ is the relevant quantity for reproducing the observed light curve and decelerations (additional contributions are not important for the present discussion). As a consequence, it is possible to provide the same fit to the observed quantities with a different luminous efficiency if the ablation coefficient is changed so that $\tau \sigma$ remains constant. From this it is clear that the reliability of the derived $\sigma$ values depends on that of $\tau$.

Figure 3 shows histograms of the ablation coefficients of sporadic (left) and shower (right) meteors. The upper panels give the distribution of $\sigma$ values for all meteors regardless of the population to which they belong. We see that the histograms for sporadic and shower meteors are similar, except for the fact that the distribution 
Table 1. Degree of success of the single body theory for the different meteoroid populations among sporadic and shower meteors. The third column gives the number $N$ of meteors belonging to the various meteoroid populations. The fourth column gives the fraction $N / N_{\text {tot }}$, with $N_{\text {tot }}$ the total number of sporadics or shower members that could be classified into one group ( $N_{\text {tot }}$ is 259 for sporadics and 85 for showers). The fifth column $\left(N_{\mathrm{sbt}}\right)$ indicates the number of meteors whose behavior is well described by the single body theory. The percentage of nonfragmenting particles $\left(N_{\mathrm{sbt}} / N\right)$ is shown in the last column. Numbers in brackets are uncertain because of poor statistics.

\begin{tabular}{llrcrr}
\hline \hline Type & Group & $N$ & $N / N_{\text {tot }}$ & $N_{\text {sbt }}$ & Percentage \\
\hline Sporadics & A & 93 & 0.36 & 41 & 44 \\
& B & 4 & 0.02 & 3 & {$[75]$} \\
& C1 & 78 & 0.30 & 70 & 90 \\
& C2 & 56 & 0.22 & 43 & 77 \\
& C3 & 16 & 0.06 & 14 & 87 \\
& D & 12 & 0.05 & 11 & 92 \\
& Total & 259 & 1.00 & 182 & 70 \\
\hline Showers & A & 0 & 0.00 & - & - \\
& B & 44 & 0.52 & 30 & 68 \\
& C1 & 27 & 0.32 & 24 & 89 \\
& C2 & 12 & 0.14 & 11 & 92 \\
& C3 & 0 & 0.00 & - & - \\
& D & 2 & 0.02 & 1 & {$[50]$} \\
& Total & 85 & 1.00 & 66 & 78 \\
\hline
\end{tabular}

is broader for the former. The mean ablation coefficients turn out to be $0.097 \pm 0.06 \mathrm{~s}^{2} \mathrm{~km}^{-2}$ for sporadics and $0.086 \pm 0.05 \mathrm{~s}^{2} \mathrm{~km}^{-2}$ for shower meteors (with uncertainties representing one standard deviations), but it is important to emphasize that they are averages over broad statistical distributions. Another important point is that the total distribution for sporadic meteors is dominated by $\mathrm{C}$-type particles. From the histograms plotted in the lower panels, we conclude that the average ablation coefficient of C-type particles is very close to $0.085 \mathrm{~s}^{2} \mathrm{~km}^{-2}$. This value seems to be appropriate for both sporadic and shower meteors, although the small number of shower members makes it difficult to draw a definite conclusion. Our average $\sigma$ value for $\mathrm{C}$-type meteoroids is in remarkable agreement with the results of Ceplecha et al. (1998). However, the distributions are slightly broader, as revealed by their standard deviations (0.04 and $0.06 \mathrm{~s}^{2} \mathrm{~km}^{-2}$ for C-type sporadic and shower meteors, respectively).

According to Fig. 3, A-type sporadic meteors are characterized by a wide range of ablation parameters. The mean $\sigma$ for these meteors is $0.14 \pm 0.11 \mathrm{~s}^{2} \mathrm{~km}^{-2}$. Ceplecha et al. (1998) quote an average ablation coefficient of only $0.04 \mathrm{~s}^{2} \mathrm{~km}^{-2}$ for A-type meteoroids, so this is an unexpected finding. Given the quality of the fits, we are confident that the distribution shown in the left central panel of Fig. 3 is correct. The discrepancy between our value and Ceplecha et al.'s value could be explained, at least in part, by the different methods used to determine them. We also want to point out that we have employed the most accurate luminous efficiencies available. Since they differ from the old ones, different ablation coefficients may
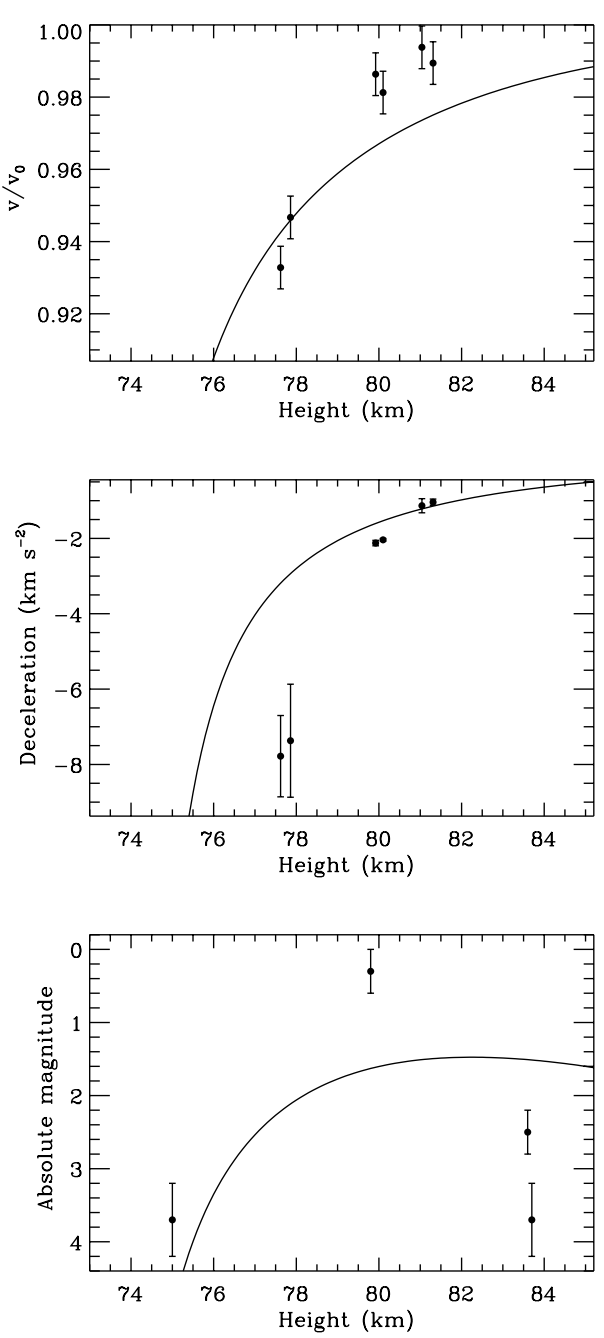

Fig. 2. Example of a faint Super-Schmidt meteor (number 4141 in JVB's catalog) for which the single body theory is not applicable. The observed decelerations are too large compared with the theoretical predictions in the later portion of the trajectory. Also, the light curve is badly reproduced, with observed magnitudes at the beginning of the trajectory being much fainter than expected. This behavior is often found when the single body theory fails.

be expected due to the dependence of $\sigma$ on $\tau$ mentioned above.

\subsection{Meteoroid bulk densities}

From the dynamical data alone we are able to determine $\sigma$ and the product $K m_{0}^{-1 / 3}$ only. The exact value of $m_{0}$ (hence of $K$ ) is obtained from the shape of the light curve. Therefore, with the precision and quantity of the data analyzed here, use of the photometric information is mandatory for reliable inferences of the shape-density coefficient. Once $K$ is known, the bulk density can be estimated as $\delta=(\Gamma A / K)^{2 / 3}$. We take $\Gamma=1$ because no significant shielding is expected to occur for the small Super-Schmidt meteors. Regarding the shape factor, we adopt $A=1.21$ on the assumption that meteoroids are spherical bodies. 

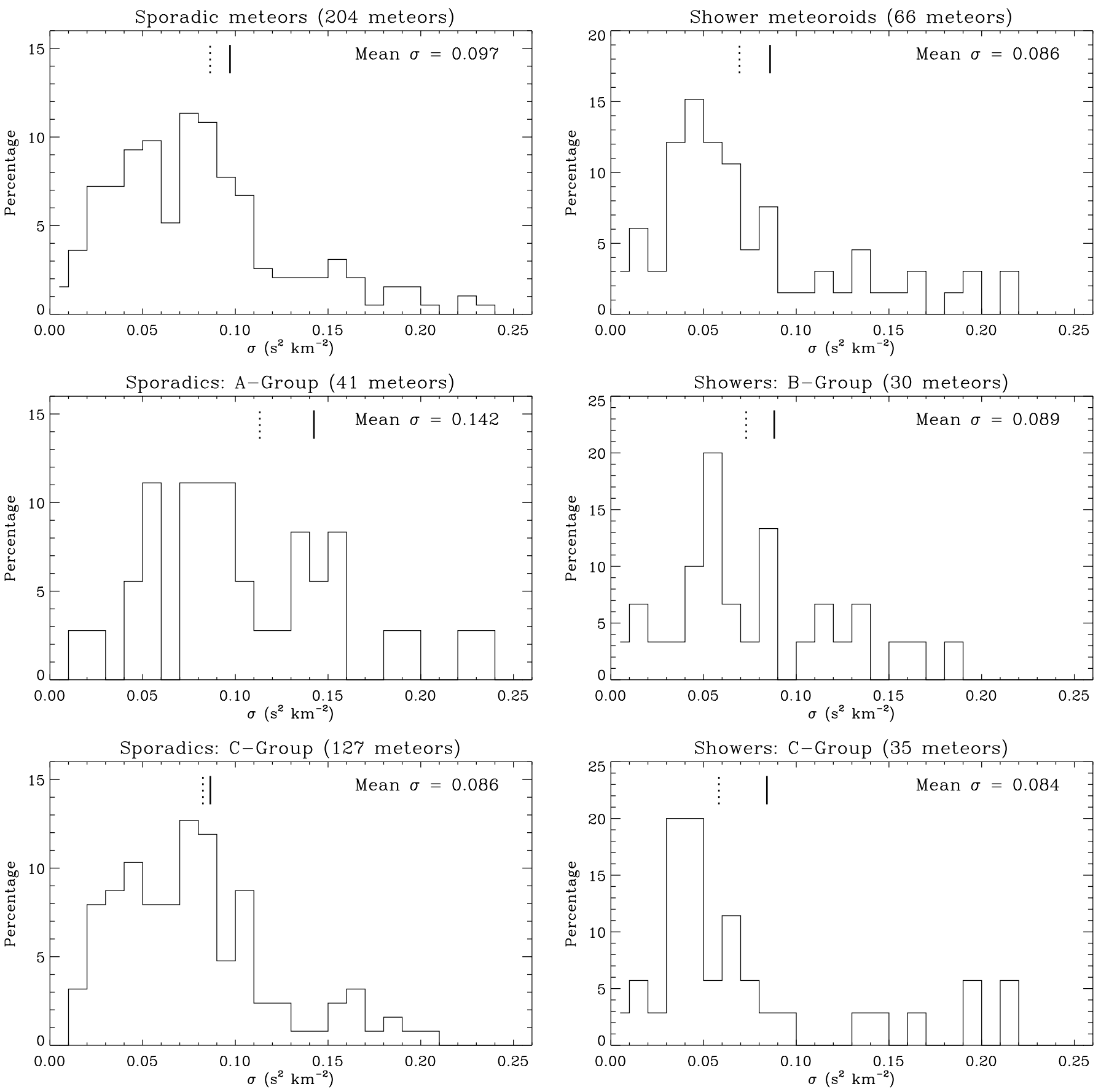

Fig. 3. Ablation coefficients from the fitting of experimental data for sporadic (left) and shower (right) meteors. Top: total sample. Middle: A-group (sporadics) and B-group (showers). Bottom: C-group meteoroids. The vertical solid and dashed lines indicate the mean and the median of each distribution, respectively.

From the arguments mentioned above it is clear that, also in this case, accurate luminous efficiencies are crucial for ensuring a correct separation of $K$ and $m_{0}$ from the fitting of the light curve.

Figure 4 shows histograms of bulk densities for the meteoroids whose dynamical and photometric behavior is well described by the single body theory. For sporadic meteors, the average density turns out to be $800 \mathrm{~kg} \mathrm{~m}^{-3}$ (upper left panel). Shower members have an average density of $900 \mathrm{~kg} \mathrm{~m}^{-3}$ (upper right panel). In both cases, individual values from 100 to $4500 \mathrm{~kg} \mathrm{~m}^{-3}$ are found, although most meteoroids are characterized by densities in the range $100-1000 \mathrm{~kg} \mathrm{~m}^{-3}$. The distributions containing all meteors are sharply peaked at $250 \mathrm{~kg} \mathrm{~m}^{-3}$, but this is due to the fact that they are dominated by C-type particles. Sporadic and shower meteors belonging to the $\mathrm{C}$ group have mean densities of $360 \mathrm{~kg} \mathrm{~m}^{-3}$ and $440 \mathrm{~kg} \mathrm{~m}^{-3}$, respectively. These values suggest that no significant 

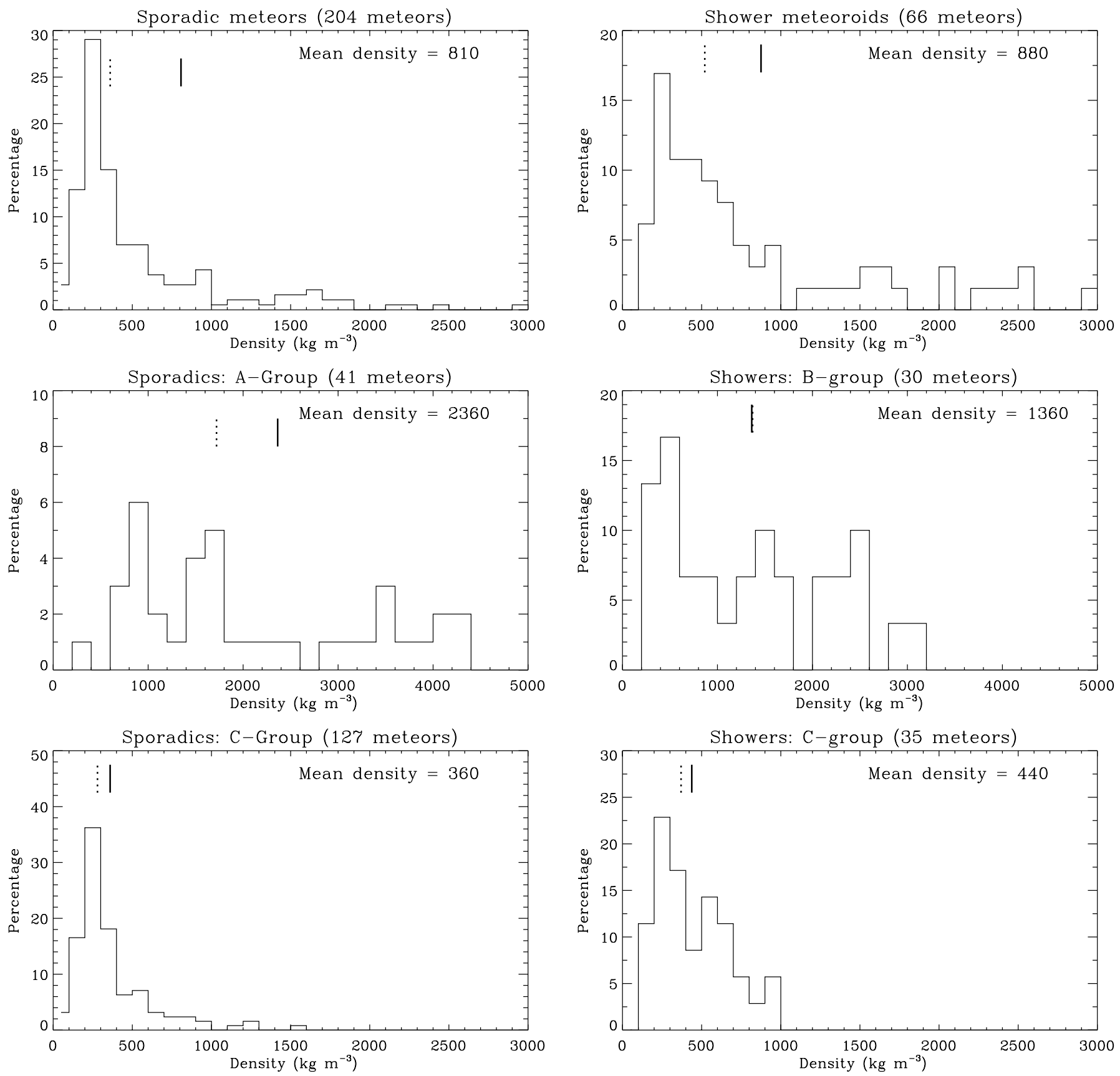

Fig. 4. Meteoroid bulk densities inferred from the fitting of experimental data for sporadic (left) and shower (right) meteors. Top: total sample. Middle: A-group (sporadics) and B-group (showers). Bottom: C-group meteoroids. The vertical solid and dashed lines indicate the mean and the median of each distribution, respectively.

differences between sporadic and shower meteoroids exist, although we cannot be more precise given the paucity of shower meteors in JVB catalog. Our mean density for Ctype objects is about half of that quoted by Ceplecha et al. (1998). A-group sporadic meteors are found to possess an average density of $2360 \mathrm{~kg} \mathrm{~m}^{-3}$, while B-group shower meteors have a mean density of $1360 \mathrm{~kg} \mathrm{~m}^{-3}$. Both values are in excellent agreement with the results of Ceplecha et al. (1998).
On average, shower meteors do not appear to be denser than sporadics. The high proportion of B-group particles among shower meteors is compensated by the larger bulk density of A-group meteoroids combined with their smaller numbers among sporadic meteors. Table 2 summarizes the mean density for different meteor showers and the range of variation of the invidivual values. Note that these figures are very uncertain because of the small sample on which they are based. Nevertheless, we find a consistent smooth transition from B-group to C-group particles and 
Table 2. Average density of shower meteors. All values are expressed in $\mathrm{kg} \mathrm{m}^{-3}$. The first column specifies the stream (Geminids, Quadrantids, Perseids, $\alpha$ Capricornids and Northern Taurids are considered). The second column indicates the meteoroid population to which the stream is ascribed. The third column gives the number of meteors used in the average. The following five columns indicate the minimum, maximum, mean, median, and standard deviation of the individual bulk densities.

\begin{tabular}{lcrcrrrr}
\hline \hline Show & Gr & $N$ & Min & Max & Mean & Median & Stdev \\
\hline GEM & B & 15 & 790 & 3120 & 1940 & 2020 & 700 \\
QUA & B & 5 & 220 & 2600 & 790 & 420 & 1000 \\
PER & C2 & 5 & 510 & 700 & 600 & 610 & 100 \\
CAP & C1 & 7 & 190 & 730 & 450 & 500 & 200 \\
NTA & C1 & 5 & 280 & 860 & 420 & 310 & 200 \\
\hline
\end{tabular}

confirm that Geminid meteoroids are among the densest shower meteors.

\section{Single body theory and quasi-continuous fragmentation}

The results presented in Sect. 5.2 cast serious doubts on the extremely large densities found by application of the QCF theory (e.g., Lebedinets 1987; Babadzhanov 1994; Novikov et al. 1996). Light curves are the basic observational material in these studies. Since the dynamical behavior is not considered in QCF analyses, the estimated densities might be erroneous if the meteoroids do not fragment during their atmospheric flight. We would like to mention here that the absence of distinct short term fluctuations in the light curves of most faint, image-intensified video meteors (Campbell et al. 1999; Murray et al. 1999; Murray et al. 1999) argues against quasi-continuous fragmentation, at least in this mass range.

Another possible explanation for the unrealistically large densities determined from QCF models is that the specific energy of fragmentation $Q_{\mathrm{f}}$ required to estimate the meteoroid bulk density from the QCF theory is significantly in error. Very few measurements of $Q_{\mathrm{f}}$ have been carried out in the laboratory, often involving materials which we do not expect in meteoroids. For these reasons, it is customary to adopt ad hoc expressions for $Q_{\mathrm{f}}$ in $\mathrm{QCF}$ analyses. A popular dependence (Lebedinets 1987) takes the form $Q_{\mathrm{f}}=10^{5}+1.8 \times 10^{2} \delta\left(\mathrm{J} \mathrm{kg}^{-1}\right)$. Here we see that the fragmentation energy is assumed to increase with $\delta$. For typical densities of $1000 \mathrm{~kg} \mathrm{~m}^{-3}, Q_{\mathrm{f}}=2.8 \times 10^{5} \mathrm{~J} \mathrm{~kg}^{-1}$, i.e., about 30 times smaller than the specific heat of ablation $\left(Q=8 \times 10^{6} \mathrm{~J} \mathrm{~kg}^{-1}\right)$. No doubt, meteoroids will always fragment with such low fragmentation energies. In practice, only when $Q_{\mathrm{f}}$ is significantly larger can the light curves be reproduced, but this requires very large meteoroid bulk densities if one adopts Lebedinets (1987) dependence. In our opinion, such an expression might not be quite justified. A hint that this is the case is the fact that classical light curves result from the QCF theory when $Q_{\mathrm{f}} \rightarrow Q$.

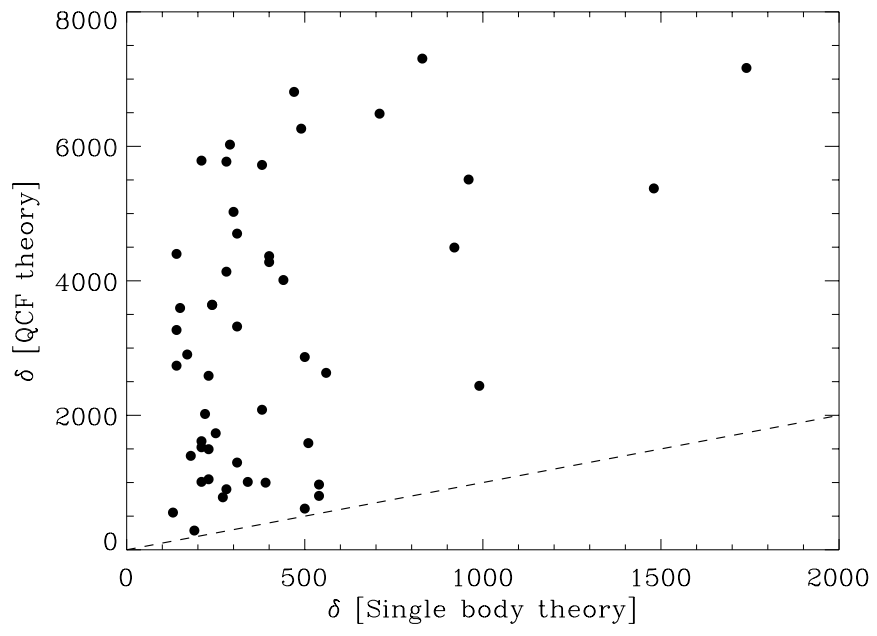

Fig. 5. Comparison of the individual meteoroid bulk densities resulting from the QCF model and the single body theory. All values are in $\mathrm{kg} \mathrm{m}^{-3}$. The dashed line indicates a one-to-one correspondence.

In order to gain more insight into this problem, the light curves of the 370 Super-Schmidt meteors considered in the previous sections have been subject to an analysis based on the QCF model. Using the expressions given by Novikov et al. (1998) we have determined the parameters $R_{0}$ and $R_{1}$ that provide the best fit to the observed magnitudes. Since the dynamical behavior is not considered, very stringent criteria were imposed to select only the best fits. In particular, the theoretical and observed magnitudes were required to agree to within $\pm 0.2 \mathrm{mag}$. As a result of our efforts we have found 59 meteors whose photometric behavior appears to be well described by the QCF model. The photometric and dynamical data of most of these 59 meteors were successfully reproduced in terms of the single body theory. We of course prefer the single-body solution because it gives a much more complete description of the overall meteor phenomenon. In any case, having 50 meteors in common makes it possible to compare the bulk densities resulting from both models. Figure 5 shows this comparison. Bulk densities for the QCF solution were derived from the best-fit $R_{0}$ parameter adopting $Q_{\mathrm{f}}=10^{5}+1.8 \times 10^{2} \delta\left(\mathrm{J} \mathrm{kg}^{-1}\right)$, and other constants as given by Novikov et al. (1998). A cursory glance at Fig. 5 demonstrates that bulk densities predicted by the QCF model are always larger than those resulting from the assumption of nonfragmenting particles, often by more than an order of magnitude. Admittedly, our QCF analysis is not very significant due to the small number of observables, but the fact that the retrieved QCF densities are similar to those found by other authors makes us confident that they are essentially correct. In a statistical sense, it is possible to reconcile the high QCF densities with the single-body values by simply using a constant $Q_{\mathrm{f}} \approx 5.5 \times 10^{6} \mathrm{~J} \mathrm{~kg}^{-1}$. This fragmentation energy is much larger than that resulting from Lebedinets expression, and 
very similar to the ablation energy. Thus, it is not surprising that the theoretical light curves remain the same with smaller bulk densities. In view of these considerations, we believe that both a critical appraisal of current estimates of fragmentation energies and an extension of the QCF theory aimed at describing the meteoroid dynamics are absolutely necessary before the QCF model can be applied to real observations with confidence. Babadzhanov (2002) has taken a step forward in this direction by choosing a fragmentation energy of $Q_{\mathrm{f}}=2 \times 10^{6} \mathrm{~J} \mathrm{~kg}^{-1}$ in his recent QCF analysis of 111 bright meteors photographed with small cameras.

\section{Discussion}

Our approach differs from previous works in a number of aspects. First, we do not make a direct comparison of dynamical and photometric masses at several points along the trajectory of the meteor, nor do we treat individual dynamical and photometric masses in a statistical way (i.e., mixing values corresponding to different meteoroids). Second, the observed velocities, decelerations and magnitudes of each individual particle are fitted simultaneously. Hence, it should come as no surprise that we find broad ranges of variation for the physical parameters of the different meteoroid populations. Instead of having sharp peaks, the distributions are wide, indicating that bodies with different ablation capabilities and densities coexist in any particular group. This does not necessarily mean that they have different chemical compositions or structure. Donn (1990) has pointed out that cometary nuclei formed by accumulation of ice/dust grains are expected to show significant internal density variations. Donn \& Meakin (1988) find that ice/dust grain mixtures may accrete at velocities of tens of $\mathrm{cm} \mathrm{s}^{-1}$ to form fractal aggregates of a few $\mathrm{mm}$ in size whose bulk density varies as $R^{-1}$, where $R$ is a characteristic size. In their model, the kinetic energy of the collisions is dissipated by the work of compression, leading to aggregates of different densities. This picture is consistent with the results of the Giotto mission to comet $1 \mathrm{P} /$ Halley. Giotto discovered that only minor parts of the nucleus surface are active, even at heliocentric distances of $1 \mathrm{AU}$ (Keller et al. 1986). The stability of the cometary surface, most of which appears to be covered by a nonvolatile material, suggests that it is formed not only by fine dust resulting from the sublimation of ices, but also by larger and denser solid particles. The existence of particles of different densities in cometary nuclei would be a natural explanation for the broad distributions found here.

Another explanation is that several subgroups may exist within the four meteoroid populations. This seems to be the case especially for A-type meteoroids. Ceplecha (1968) showed that A-group particles span a wide area in the eccentricity-perihelium distance diagram. In fact, up to 10 statistical subgroups within the A group were recognized. This implies that we are dealing with bodies of different orbital characteristics. Particles having small perihelium distances get more heated than those with larger perihelium distances. This will cause the volatile species to be highly depleted or even completely lost. In addition, meteoroids are exposed to space conditions during different periods of time. As a result, the collisional and cosmic ray irradiation histories will be different for each particle, and one can speculate that these processes may alter the original bulk densities. In summary, a wide range of ablation parameters and densities is not unreasonable given the different orbital characteristics of the individual meteoroids.

It should be clear by now that care must be exercized when quoting or interpreting mean values, as they normally represent averages over irregular, broad statistical distributions. In this work, the mean densities for the different meteoroid populations have been found to be approximately $2400 \mathrm{~kg} \mathrm{~m}^{-3}$ (A group), $1400 \mathrm{~kg} \mathrm{~m}^{-3}$ (B group) and $400 \mathrm{~kg} \mathrm{~m}^{-3}$ (C group). Keeping in mind the above considerations, the degree of coincidence with Ceplecha's et al. (1998) values is very satisfactory. The only significant departure occurs for C-type meteoroids, to which we ascribe half of the density suggested by these authors.

In order to gauge the significance of this agreement we want to point out that the average densities found by Ceplecha (1988) were derived on completely different grounds. They are based on the fact that the $K_{\mathrm{B}}$ parameter (Eq. (1)) is a good measure of the product of the heat conductivity, density and specific heat of the meteoroid (Ceplecha 1967). Thus, an absolute calibration of the $K_{\mathrm{B}}$ scale (together with several theoretical assumptions) makes it possible to estimate meteoroid bulk densities from the observed $K_{\mathrm{B}}$ values. Ceplecha (1967) carried out such a calibration with the help of the beginning heights of three meteorites whose densities could be measured in the laboratory (Pribram, Lost City, and Innisfree). It is important to remark here that these fireballs were much brighter than typical Super-Schmidt meteors. The advantage of using the observed $K_{\mathrm{B}}$ values for assigning mean physical properties is that fragmentation (should it occur) cannot lead to wrong results because it has a minor influence on the earlier parts of the trajectory. Indeed, the derived densities would apply to both fragmenting and nonfragmenting objects.

Our results are consistent with the idea that fragmentation does not have a significant impact on the dynamical and photometric behavior of faint Super-Schmidt meteors (i.e., of particles in the mass range from $10^{-5}$ to $10^{-2} \mathrm{~kg}$ ). The situation may be different for other mass ranges. It seems established that fragmentation is mainly a surface phenomenon for the big particles producing fireballs and small-camera meteors. As such, fragmentation has a minor influence on the observed quantities, and the single body theory is appropriate (e.g. McCrosky \& Ceplecha 1970). Bulk densities similar to those reported here are inferred for these objects. At the lower mass end, there is evidence from image-intensified video observations that 
very faint meteors are better described in terms of the dustball model of Hawkes \& Jones (1975). Probably, such small particles are collections of tiny grains held together by a more volatile glue component. The absence of wake (Shadbolt \& Hawkes 1995) and the weak dependence of beginning and end heights on mass (Hawkes \& Jones 1975; Campbell et al. 2000) suggest that the glue completely evaporates prior to the onset of light production, releasing a swarm of individual grains. These grains behave as independent particles which ablate and emit light according to the single body theory. Due to their small sizes, they quickly slow down because of collisions with air molecules. From an analysis of the observed beginning heights of Leonid meteoroids, Campbell et al. (2000) came to the conclusion that iron and solid stone are not the main constituents of the fundamental grains. Among other compositions, these authors suggest organic compounds or very porous stony material having bulk densities lower than $3500 \mathrm{~kg} \mathrm{~m}^{-3}$. The dustball model of Hawkes \& Jones was developed to explain the ablation trajectories of meteoroids of a variety of sizes with a single physical structure. Very small meteoroids are thought to behave as described earlier. For the brighter meteors, the dustball model may result in light curves and trajectories similar to those of compact bodies, depending on the thermal properties of the glue (which determine when grain release occurs). In this regard, our finding that a significant fraction of the Super-Schmidt meteors behave as nonfragmenting particles (possibly with a different value of the ablation parameter) is not in conflict with the model of Hawkes \& Jones.

A final word concerning the interpretation of our results is in order. Strictly speaking, we cannot rule out that the Super-Schmidt meteoroids conforming to the single body theory actually underwent some kind of fragmentation. If the parent body breaks into $N$ separate fragments, for example, the observed deceleration would be that corresponding to one of the pieces, whereas all fragments would contribute to the light curve. In this situation, the single body theory would able to provide a fit to the observations, but the inferred bulk densities would be smaller than the actual value by a factor $N^{-1 / 2}$. If this is the case, the low densities found from application of the single body model would not be real. We cannot discard this scenario completely, but McCrosky \& Ceplecha (1970) have given arguments against it. The basic idea is that, in order to explain the large variations of the shape-density coefficient of individual meteoroids assuming a bulk density of $\sim 3500 \mathrm{~kg} \mathrm{~m}^{-3}$, all meteors must fragment into a large number of pieces (for C-type particles, the required number varies between roughly 10 and 1500, independently of the meteoroid mass). In addition, it is necessary that they all have the same sizes, because otherwise the fragments would be subject to differential aerodynamic lags and this is not observed. McCrosky \& Ceplecha (1970) find these requirements unrealistic and conclude that most meteoroids are probably low density objects.

\section{Conclusions}

A numerical procedure implementing the single body theory has been developed in order to reproduce the dynamical and photometric behavior of meteoroids in the Earth's atmosphere. It has been used to determine the ablation coefficients, shape-density coefficients and initial masses of 370 faint Super-Schmidt meteors. 270 of these meteors are described reasonably well as nonfragmenting particles. In general, velocities and decelerations are almost perfectly reproduced. Matching the light curve is somewhat more difficult, but very important for constraining the value of the initial mass (hence of the shape-density coefficient $K$, since from dynamical data alone we are able to determine precise values of $K m_{0}^{-1 / 3}$ only).

From the inferred $K$ parameters we have estimated meteoroid bulk densities on the assumption of spherical shapes and free molecular flow. Sporadic and shower meteoroids have been found to possess a mean density of 800 and $900 \mathrm{~kg} \mathrm{~m}^{-3}$, respectively. The distribution of individual values are broad, indicating that objects of different characteristics (but probably of the same composition and structure) coexist within the same meteoroid population. For A-type, B-type and C-type meteoroids we find average densities of 2400,1400 and $400 \mathrm{~kg} \mathrm{~m}^{-3}$, in general agreement with the results of independent methods (Ceplecha et al. 1998). Among shower meteoroids, Geminids are the densest objects (mean density of about $\left.2000 \mathrm{~kg} \mathrm{~m}^{-3}\right)$.

We do not confirm the high bulk densities resulting from quasicontinuous fragmentation (QCF) models. By applying the QCF theory to the same photometric data, we have shown that too small fragmentation energies and limitations of the theory in modeling the dynamical behavior of the meteoroids are likely causes of the discrepancy. The QCF bulk densities can be brought to an agreement with the single body values if the actual fragmentation energy is close to $5.5 \times 10^{6} \mathrm{~J} \mathrm{~kg}^{-1}$, although large differences cannot be removed for many individual meteors.

No doubt, the most important result of this work is that the ablation of solid, compact particles explains the behavior of a significant fraction (73\%) of faint SuperSchmidt meteors. Apparently, these objects did not undergo severe fragmentation processes as this would have caused the single body theory to fail. For the remaining $28 \%$ of the sample, however, more complex models appear to be necessary. This highlights the importance of additional theoretical developments and new observations in order to improve our understanding of the structure of meteoroids and their interaction with the Earth's atmosphere.

Acknowledgements. We would like to express our gratitude to Dr. Z. Ceplecha for motivating this investigation and his help in numerous subjects. We also thank the referee, Dr. R. Hawkes, for very detailed comments and suggestions. This work has been partially funded by the Spanish Ministerio de Ciencia y Tecnología under project AYA2001-4009E. 


\section{References}

Babadzhanov, P. B. 1992, Sol. Syst. Res., 26, 488

Babadzhanov, P. B. 1994, in Proc. IAU Symp. 160, Asteroids, Comets, Meteors 1993, ed. A. Milani, M. Di Martino, A. Cellino (Kluwer, Dordrecht), 45

Babadzhanov, P. B. 2002, A\&A, 384, 317

Babadzhanov, P. B., Novikov, G. G., Lebedinets, V. N., \& Blokhin, A. V. 1988, Sol. Syst. Res., 22, 51

Bellot Rubio, L. R., Martínez González, M. J., Ruiz Herrera, L., et al. 2001, in Proc. Meteoroids 2001 Conference, ESASP, 495, 525

Bronshten, V. A. 1983, Physics of Meteoric Phenomena (D. Reidel Publishing Company, Dordrecht)

Campbell, M., Hawkes, R. L., \& Babcock, D. 1999, in Meteoroids 1998, ed. W. J. Baggaley, \& V. Porubcan, 363

Campbell, M. D., Brown, P. G., LeBlanc, A. G., et al. 2000, Meteorit. Planet. Sci., 35, 1259

Ceplecha, Z. 1958, Bull. Astron. Inst. Czechosl., 9, 154

Ceplecha, Z. 1966, Bull. Astron. Inst. Czechosl., 17, 221

Ceplecha, Z. 1967, Smithson. Contr. Astrophys., 11, 35

Ceplecha, Z. 1968, SAO Special Report, 279, 1

Ceplecha, Z. 1988, Bull. Astron. Inst. Czechosl., 39, 221

Ceplecha, Z., Borovicka, J., \& Spurný, P. 2000, A\&A, 357, 1115

Ceplecha, Z., Borovicka, J., Elford, W. G., et al. 1998, Space Sci. Rev., 84, 327

Donn, B. D. 1990, A\&A, 235, 441

Donn, B. D., \& Meakin, P. 1988, BAAS, 20, 840

Jones, J., \& Kaiser, T. R. 1966, MNRAS, 133, 411

Hawkes, R. L., \& Jones, J. 1975, MNRAS, 173, 339

Hedin, A. E. 1991, J. Geophys. Res., 96, 1159

Fisher, A. A., Hawkes, R. L., Murray, I. S., Campbell, M. D., \& Leblanc, A. G. 2000, Planet. Space Sci., 48, 911
Jacchia, L. G. 1955, ApJ, 121, 521

Jacchia, L. G. 1958, Smithson. Contr. Astrophys., 2, 181

Jacchia, L. G., Verniani, F., \& Briggs, R. E. 1967, Smithson. Contr. Astrophys., 10, 1 (JVB)

Keller, C. U., Arpigny, C., Barbieri, C., et al. 1986, Nature, 321,320

Lebedinets, V. N. 1987, Sol. Syst. Res., 21, 41

McCrosky, R. E., \& Ceplecha, Z. 1970, Bull. Astron. Inst. Czechosl., 21, 271

McCrosky, R. E., \& Posen, A. 1961, Smithson. Contr. Astrophys., 4, 15

Murray, I. S., Beech, M., Taylor, M. J., Jenniskens, P., \& Hawkes, R. L. 2000, Earth, Moon and Planets, 82-83, 351

Murray, I. S., Hawkes, R. L., \& Jenniskens, P. 1999, Meteorit. Planet. Sci., 34, 949

Novikov, G. G., Lebedinets, V. N., \& Blokhin, A. V. 1984, SvA Lett., 10, 27

Novikov, G. G., Pecina, P., \& Konovalova, N. A. 1996, A\&A, 306, 991

Novikov, G. G., Pecina, P., \& Konovalova, N. A. 1998, A\&A, 329,769

Press, W. H., Flannery, B. P., Teukolsky, S. A., \& Vetterling, W. T. 1986, Numerical Recipes-The Art of Scientific Computing (Cambridge University Press, Cambridge)

ReVelle, D., \& Ceplecha, Z. 2001, in Proc. Meteoroids 2001 Conference, ESA-SP, 495, 507

Shadbolt, L., \& Hawkes, R. L. 1995, Earth Moon Planets, 68, 493

Verniani, F. 1965, Smith. Contr. Astrophys., 8, 141

von Zahn, U., Gerding, M., Hoffner, J., McNeil, W. J., \& Murad, E. 1999, Meteorit. Planet. Sci., 34, 1017 